\title{
LHC limits on gluinos and squarks in the minimal Dirac gaugino model.
}

\section{Humberto Reyes-González ${ }^{* a}$}

${ }^{a}$ Laboratoire de Physique Subatomique et de Cosmologie, Université Grenoble-Alpes, CNRS/IN2P3, 53 Avenue des Martyrs, F-38026 Grenoble, France.

E-mail: gonzalezelpsc.in2p3.fr

\begin{abstract}
Most SUSY searches at the LHC are optimised for the MSSM, where gauginos are Majorana particles. By introducing Dirac gauginos, we obtain an enriched phenomenology, from which considerable differences in the LHC signatures and limits are expected as compared to the MSSM. Concretely, in the minimal Dirac gaugino model (MDGSSM) we have six neutralino and three chargino states. Moreover, production cross sections are enhanced for gluinos, while for squarks they are suppressed. In this contribution we show concrete results for the current LHC limits on gluinos and squarks in this model.
\end{abstract}

ALPS 2019 An Alpine LHC Physics Summit

April 22 - 27, 2019

Obergurgl, Austria

${ }^{*}$ Speaker. 


\section{Introduction.}

Originally proposed to allow the gluino to be massive [1], models with Dirac Gauginos (DG) have interesting features such as providing a tree level boost to the Higgs mass, being able to be associated to $N=2$ supersymmetry, posibility of preserving R-symmetry and increase naturalness. See [2], for a concise review on Dirac Gauginos.

\begin{tabular}{|c|c|c|c|c|c|}
\hline Names & & Spin 0 & Spin 1/2 & Spin 1 & $S U(3), S U(2), U(1)_{Y}$ \\
\hline Quarks & $\mathbf{Q}$ & $\tilde{Q}=\left(\tilde{u}_{L}, \tilde{d}_{L}\right)$ & $\left(u_{L}, d_{L}\right)$ & & $(\mathbf{3}, \mathbf{2}, 1 / 6)$ \\
& $\mathbf{u}^{\mathbf{c}}$ & $\tilde{u}_{L}^{c}$ & $u_{L}^{c}$ & & $(\overline{\mathbf{3}}, \mathbf{1},-2 / 3)$ \\
$(\times 3$ families $)$ & $\mathbf{d}^{\mathbf{c}}$ & $\tilde{d}_{L}^{c}$ & $u_{L}^{c}$ & & $(\overline{\mathbf{3}}, \mathbf{1}, 1 / 3)$ \\
\hline Leptons & $\mathbf{L}$ & $\left(\tilde{v}_{e L}, \tilde{e}_{L}\right)$ & $\left(v_{e L}, e_{L}\right)$ & & $(\mathbf{1}, \mathbf{2},-1 / 2)$ \\
$(\times 3$ families $)$ & $\mathbf{e}^{\mathbf{c}}$ & $\tilde{e}_{L}^{c}$ & $e_{L}^{c}$ & & $(\mathbf{1}, \mathbf{1}, 1)$ \\
\hline Higgs & $\mathbf{H}_{\mathbf{u}}$ & $\left(H_{u}^{+}, H_{u}^{0}\right)$ & $\left(\tilde{H}_{u}^{+}, \tilde{H}_{u}^{0}\right)$ & & $(\mathbf{1}, \mathbf{2}, 1 / 2)$ \\
& $\mathbf{H}_{\mathbf{d}}$ & $\left(H_{d}^{0}, H_{d}^{-}\right)$ & $\left(\tilde{H}_{d}^{0}, \tilde{H}_{d}^{-}\right)$ & & $(\mathbf{1}, \mathbf{2},-1 / 2)$ \\
\hline Gluons & $\mathbf{W}_{\mathbf{3} \alpha}$ & & $\tilde{g}_{\alpha}$ & $g$ & $\mathbf{( 8 , 1 , 0 )}$ \\
W & $\mathbf{W}_{\mathbf{2} \alpha}$ & & $\tilde{W}^{ \pm}, \tilde{W}^{0}$ & $W^{ \pm}, W^{0}$ & $\mathbf{( 1 , 3}, 0)$ \\
B & $\mathbf{W}_{\mathbf{1} \alpha}$ & & $\tilde{B}^{\prime}$ & $B$ & $(\mathbf{1}, \mathbf{1}, 0)$ \\
\hline \hline DG-octet & $\mathbf{O}_{\mathbf{g}}$ & $O_{g}$ & $\tilde{g}^{\prime}$ & & $(\mathbf{8}, \mathbf{1}, 0)$ \\
DG-triplet & $\mathbf{T}$ & $\left\{T^{0}, T^{ \pm}\right\}$ & $\left\{\tilde{W}^{\prime \pm}, \tilde{W}^{\prime 0}\right\}$ & & $(\mathbf{1}, 3,0)$ \\
DG-singlet & $\mathbf{S}$ & $S$ & $\tilde{B}^{\prime}$ & & $(\mathbf{1}, \mathbf{1}, 0)$ \\
\hline
\end{tabular}

Table 1: Chiral and gauge multiplet fields in the model. The red coloured section corresponds to the new chiral multiplets that completes the DG model. The rest, are the usual multiplets found in the MSSM.

To introduce Dirac masses for the gauginos, we need to add a Weyl fermion in the adjoint representation of each gauge group; these are embedded in chiral superfields $\mathbf{S}, \mathbf{T}, \mathbf{O}$ which are respectively a singlet, triplet and octet, and carry zero R-charge. The resulting field content is summarised in Table 1. The singlet and triplet fields can have new superpotential couplings with the Higgs,

$$
W \supset \lambda_{S} \mathbf{S} \mathbf{H}_{\mathbf{u}} \cdot \mathbf{H}_{\mathbf{d}}+2 \lambda_{T} \mathbf{H}_{\mathbf{d}} \cdot \mathbf{T} \mathbf{H}_{\mathbf{u}}
$$

In DG models, different limits from collider searches can be expected when looking at the production cross sections of squarks and gluinos. For squarks, pair production is suppressed due to the absence of a chirality flip (see Fig. 1), whereas gluino-pair production is enhanced, because of an enlarged number of degrees of freedom compared to the MSSM. We can also expect different limits from the fact that DG models have a more complex electroweak-ino spectrum, with six neutralino and three chargino mass eigenstates (as compared to four and two, respectively, in the MSSM), which may appear in gluino and squark cascade decays.

In the following, we show limits to the masses of the gluinos and squarks in the MDGSSM obtained from reintepreting LHC results. A complete description of this work can be found in [3]. 
Squark production, LHC $13 \mathrm{TeV}, \mathrm{m}_{\widetilde{\mathrm{q}}}=1.5 \mathrm{TeV}$.

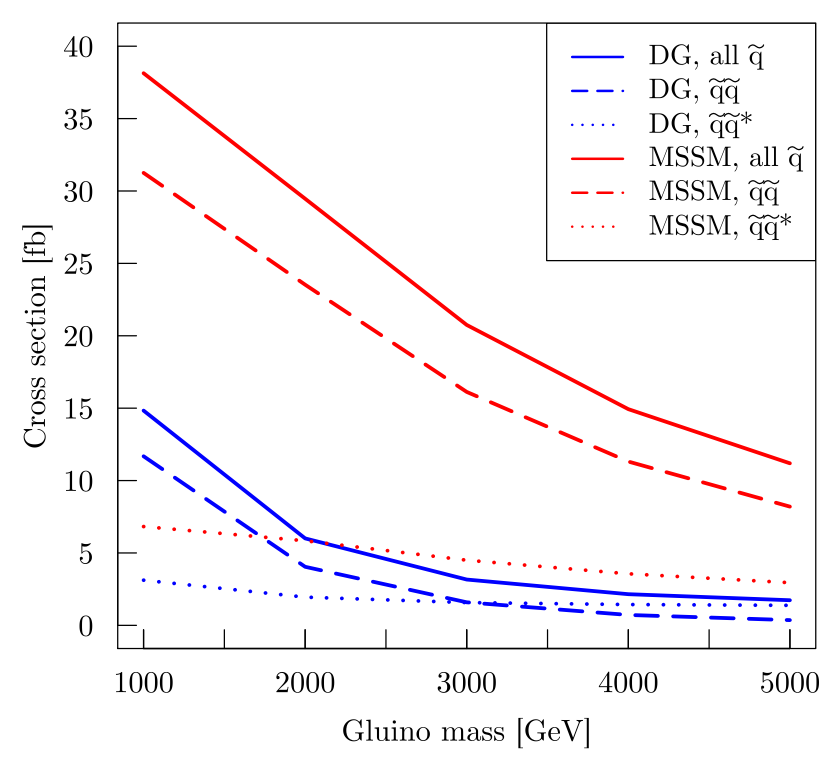

Figure 1: Squark production cross-sections at leading order (LO) for the $13 \mathrm{TeV}$ LHC as a function of the gluino mass in the MSSM (in red) and in the DG case (in blue).

\section{Deriving limits on the masses of gluinos and squarks.}

In this contribution, we will focus on three benchmark scenarios, denoted as DG1, DG2 and DG3. In all of them, $m_{D Y}=200 \mathrm{GeV}, \mu=400 \mathrm{GeV}$ and $\tan \beta=2, m_{D 2}=500 \mathrm{GeV}$ and $\lambda_{T}=2$, where $m_{D Y}$ and $m_{D Y}$ are the soft mass terms of the Dirac binos and winos, respectively. This translates into a hierarchical spectrum of bino $\left(\tilde{\chi}_{1,2}^{0}\right)$-, higgsino $\left(\tilde{\chi}_{3,4}^{0}\right)$ - and wino $\left(\tilde{\chi}_{5,6}^{0}\right)$-like states with masses of about 200,400 and $500 \mathrm{GeV}$, respectively.

With this configuration $\tilde{\chi}_{1,2}^{0}$, will be mostly bino states with a mass splitting given by $m_{\tilde{\chi}_{2}^{0}}-$ $m_{\tilde{\chi}_{1}^{0}}=\frac{M_{Z}^{2} \sin \theta_{W}}{\mu} \frac{2 \lambda_{S}^{2}-g_{Y}^{2}}{g_{Y}^{2}} \sin 2 \beta$. The influence of $\lambda_{S}$ on the mass splitting of the bino like states and, subsequently, on the mean decay length of $\tilde{\chi}_{2}^{0}$, is shown in Fig. 2; this was important for choosing our benchmark scenarios. When $\left|\lambda_{S}\right|$ is small, the mass difference is on the MeV range, and the mean decay length of $\tilde{\chi}_{2}^{0}$ can be of the order of kilometers, so it will appear as a co-LSP. With this in mind, we have chosen $\lambda_{S}=-.27$, for the DG1 scenario. When $\left|\lambda_{S}\right|$ is large, the mean decay length $\tilde{\chi}_{2}^{0}$ is so small that it decays promptly; this is the case in DG2 and DG3, where $\lambda_{S}=-.74$.

Finally, the masses of gluinos and squarks are treated as free parameters, while the masses of the $3 r d$ generation squarks are adjusted such that $m_{h_{1}} \in[123,127] \mathrm{GeV}$. The rest of the particle content is decoupled.

As a first step, we applied the Simplified Model Limit approach using SModels [4]. The result for the DG1 scenario, is shown in Fig. 3. When $m_{\tilde{g}}<m_{\tilde{q}}$ the strongest constraint comes from the $p p \rightarrow \tilde{g} \tilde{g}, \tilde{g} \rightarrow q \bar{q} \tilde{\chi}_{1}^{0}$ simplified model (denoted as T1) and excludes gluino masses up to about $1250 \mathrm{GeV}$ for LO cross-sections. When $m_{\tilde{q}}<m_{\tilde{g}}$, the strongest constraint mostly comes from the $p p \rightarrow \tilde{q} \tilde{q}^{(*)}, \tilde{q} \rightarrow q \tilde{\chi}_{1}^{0}$ simplified model (denoted as T2), excluding squark masses up to roughly 

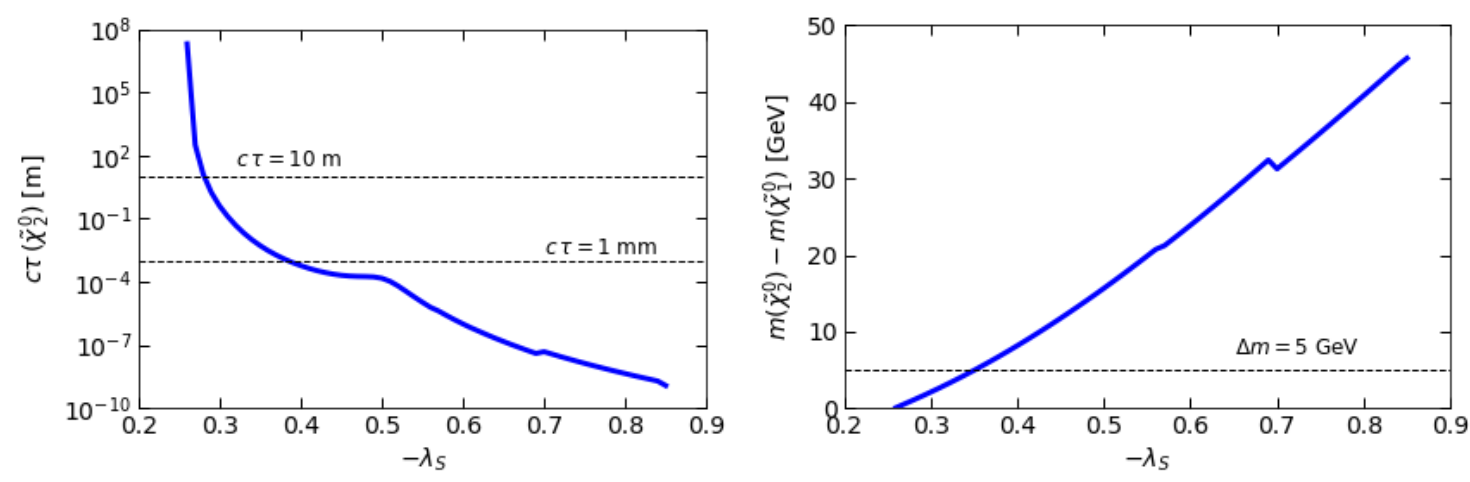

Figure 2: Influence of $\lambda_{S}$ on the mass splitting between the two bino-like mass eigenstates $\tilde{\chi}_{1,2}^{0}$ (left) and on the lifetime of the $\tilde{\chi}_{2}^{0}$ (right) .

$1300 \mathrm{GeV}$ as long as the gluino is not too heavy.

We have to keep in mind, however, that the constraints which can be derived in the context of simplified models considerably weaken in realistic scenarios where the gluinos (squarks) share out their branching ratios over several decay channels [5]. For instance, in the DG1 scenario $\operatorname{BR}\left(\tilde{g} \rightarrow q \bar{q} \tilde{\chi}_{1}^{0}\right) \backsim 0.1$, which means that only $1 \%$ of the total gluino-pair production is constrained by the $p p \rightarrow \tilde{g} \tilde{g}, \tilde{g} \rightarrow q \bar{q} \tilde{\chi}_{1}^{0}$ simplified model. This clearly suggests that the simplified model limits are not sufficient for constraining complex scenarios as the ones considered here. Nonetheless, this approach provides a very fast way to derive results that can serve as lower bounds, when scanning for the true exclusion lines of such cases. To obtain more realistic constraints, a full recasting of

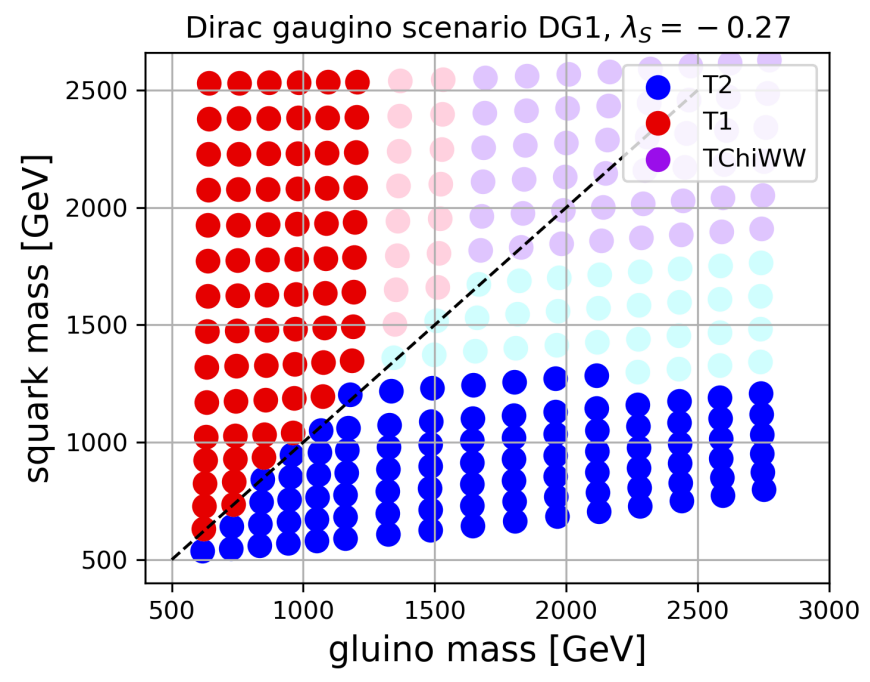

Figure 3: SModelS constraints in the gluino versus squark mass plane, for DG1. The colour code denotes the simplified model which gives the strongest constraint (T1: $p p \rightarrow \tilde{g} \tilde{g}, \tilde{g} \rightarrow q \bar{q} \tilde{\chi}_{1}^{0} ; \mathrm{T} 2: p p \rightarrow \tilde{q} \tilde{q}^{(*)}$, $\tilde{q} \rightarrow q \tilde{\chi}_{1}^{0}$; TChiWW: $p p \rightarrow \tilde{\chi}_{i}^{ \pm} \tilde{\chi}_{i}^{ \pm}, \tilde{\chi}_{i}^{ \pm} \rightarrow W^{ \pm} \tilde{\chi}_{1}^{0}$ ). Full-colour (non-transparent) points are excluded by SModelS, while light-shaded points escape the simplified model limits.

experimental searches should be performed. With this aim, we implemented (see [6]) the 'Meff- 
based search' signal regions of the ATLAS multijet search [7] for squarks and gluinos in final states with 2-6 jets and large missing transverse momentum, using $36 \mathrm{fb}^{-1}$ of $\sqrt{s}=13 \mathrm{TeV} p p$ collision data, in MadAnalysis 5 [8].

We scanned over gluino and light-flavor squark masses for the DG1 DG2 and DG3 scenarios. For each scan point, we simulated 30K events with MadGraph5_aMC@NLO [9], parton shower and hadronization were done in Pythia 8.2 [10] and the simulation of the ATLAS detector with Delphes 3 [11]. Afterwards, the events were analysed with MadAnalysis 5 and exclusion confidence levels (CL) were computed. Finally, for limit setting, since signal regions are inclusive, only the "best" (i.e. the statistically most sensitive) was used.

Figure 4 shows the $95 \%$ CL exclusion lines in the gluino vs. squark mass plane for the three benchmark scenarios and for MSSM1, an MSSM scenario equivalent to DG1. In the region $m_{\tilde{q}}>$ $m_{\tilde{g}}$ we found a robust limit of $m_{\tilde{g}} \gtrsim 1.65 \mathrm{TeV}$ when squarks are very heavy, in all cases. In the region $m_{\tilde{g}}>m_{\tilde{q}}$, for DG2 and DG3 we obtained a squark limit of $m_{\tilde{q}} \gtrsim 1.1 \mathrm{TeV}$, while for DG1 the limit reaches $m_{\tilde{q}} \gtrsim 1.4 \mathrm{TeV}$. The difference is a consequence of the $\tilde{\chi}_{2}^{0} \rightarrow Z^{*} \tilde{\chi}_{1}^{0}$ decays, which are present in DG3 and DG2, but not in DG1.

We also observe different "dips" in the exclusion contours for the different benchmark scenarios which originate from a switch of the best signal region from $6 \mathrm{j}-$ Meff-1800 (6 jets, $M_{\text {eff }}>$ $1800 \mathrm{GeV}$ ) to $6 \mathrm{j}$-Meff-2600 (6 jets, $M_{\text {eff }}>2600 \mathrm{GeV}$ ) at different, but close, values of gluino mass for each scenario. Finally, looking at the exclusion line for MSSM1, we see the expected

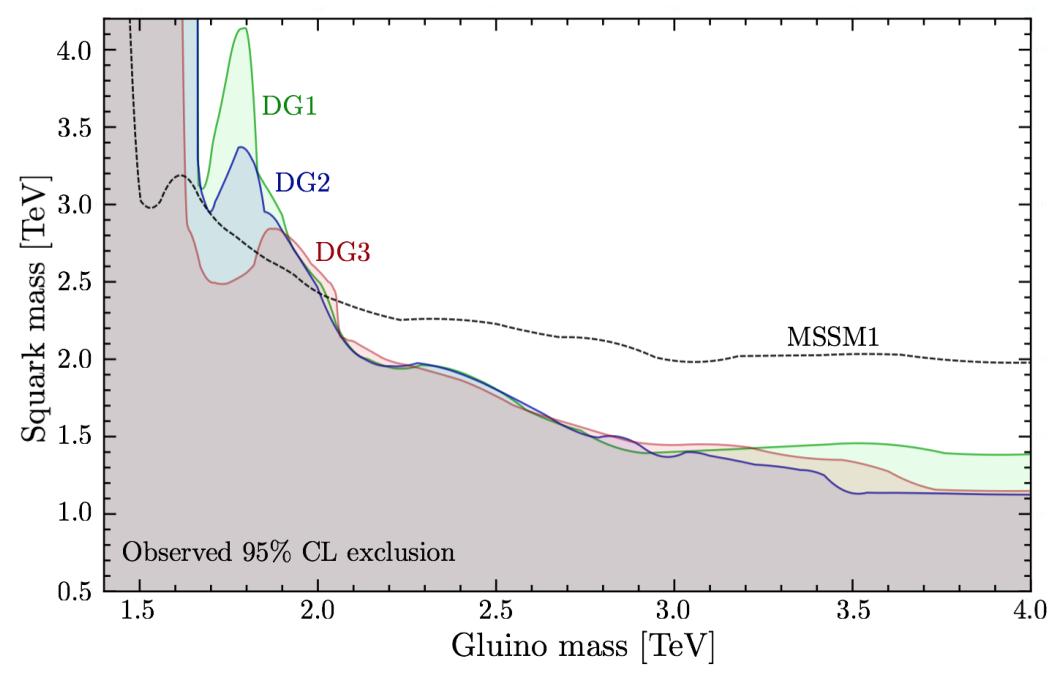

Figure 4: 95\% CL exclusion limits in the gluino vs. squark mass plane for DG1 (green), DG2 (blue) and DG3 (red) contrasted with MSSM1 (black dashed line), derived from the recasting of [7].

$\sim 200 \mathrm{GeV}$ lower gluino mass limit for an MSSM scenario and a stronger squark mass limit when gluinos are heavy, still reaching $m_{\tilde{q}} \gtrsim 2 \mathrm{TeV}$ for $4 \mathrm{TeV}$ gluinos, as Majorana gluinos decouple very slowly.

\section{Conclusions.}

As expected, by allowing Dirac gauginos we obtained LHC limits that differ from the ones of the MSSM. The differences found come mainly from suppressed squark production, enhanced 
gluino production and a more complicated electroweakino spectrum. Also interesting is the possibility of a long lived $\tilde{\chi}_{2}^{0}$; a follow-up project aims to study this kind of scenarios, in the light of Long Lived Particle searches.

In general, we think that studying the MDGSSM and other non-minimal SUSY models is a well motivated endeavor, and we hope that this contribution provides a good example of its usefulness.

\section{Acknowledgements.}

The work presented here has been carried out in collaboration with G. Chalons, M. Goodsell, S. Kraml, and S. Williamson. H. Reyes-González is funded by Consejo Nacional de Ciencia y Tecnología, CONACyT, scholarship no. 291169. He also acknowledges support from the IN2P3 project "Théorie-LHCiTools" for attending this conference and thanks S. Kraml fo her comments on the manuscript.

\section{References}

[1] P. Fayet, Massive Gluinos, Phys. Lett. 78B (1978) 417.

[2] K. Benakli, Dirac Gauginos: A User Manual, Fortsch. Phys. 59 (2011) 1079 [1106. 1649 ].

[3] G. Chalons, M. D. Goodsell, S. Kraml, H. Reyes-González and S. L. Williamson, LHC limits on gluinos and squarks in the minimal Dirac gaugino model, JHEP 04 (2019) 113 [1812. 09293 ].

[4] F. Ambrogi, S. Kraml, S. Kulkarni, U. Laa, A. Lessa, V. Magerl et al., SModelS v1.1 user manual, Comput. Phys. Comm. 227 (2018) 72 [1701.06586].

[5] F. Ambrogi, S. Kraml, S. Kulkarni, U. Laa, A. Lessa and W. Waltenberger, On the coverage of the pMSSM by simplified model results, Eur. Phys. J. C78 (2018) 215 [1707.09036].

[6] G. Chalons and H. Reyes-Gonzalez, MadAnalysis 5 implementation of ATLAS-SUSY-16-07 (arXiv:1712.02332), 2018. 10.7484/INSPIREHEP.DATA.56DC.PPE2.

[7] ATLAS collaboration, Search for squarks and gluinos in final states with jets and missing transverse momentum using 36/fb of sqrt(s)=13 TeV pp collision data with the ATLAS detector, Phys. Rev. D97 (2018) 112001 [1712.02332].

[8] B. Dumont, B. Fuks, S. Kraml, S. Bein, G. Chalons, E. Conte et al., Toward a public analysis database for LHC new physics searches using MADANALYSIS 5, Eur. Phys. J. C75 (2015) 56 [1407.3278].

[9] J. Alwall, R. Frederix, S. Frixione, V. Hirschi, F. Maltoni, O. Mattelaer et al., The automated computation of tree-level and next-to-leading order differential cross sections, and their matching to parton shower simulations, JHEP 07 (2014) 079 [1405.0301].

[10] T. Sjöstrand, S. Ask, J. R. Christiansen, R. Corke, N. Desai, P. Ilten et al., An Introduction to PYTHIA 8.2, Comput. Phys. Commun. 191 (2015) 159 [1410.3012].

[11] DELPHES 3 collaboration, DELPHES 3, A modular framework for fast simulation of a generic collider experiment, JHEP 02 (2014) 057 [1307. 6346]. 